\title{
An extension of the Golden-Thompson theorem
}

Hongyi Li and Di Zhao*

\section{"Correspondence:} zdhyl2010@163.com

School of Mathematics and System Science, Beihang University, Beijing, 100191, China

\author{
Abstract \\ In this paper, we shall prove $\left|\operatorname{tr} e^{A+B}\right| \leq \operatorname{tr}\left(\left|e^{A}\right|\left|e^{B}\right|\right)$ for normal matrices $A, B$. In \\ particular, $\operatorname{tr} e^{A+B} \leq \operatorname{tr}\left(e^{A} e^{B}\right)$ if $A, B$ are Hermitian matrices, yielding the \\ Golden-Thompson inequality. \\ MSC: $15 \mathrm{~A} 16 ; 47 \mathrm{~A} 63 ; 15 \mathrm{~A} 45$
}

Keywords: normal matrix; majorization; Golden-Thompson inequality

\section{Introduction and preliminaries}

The famous Golden-Thompson inequality [1-4] for Hermitian matrices $A, B$ states that $\operatorname{tr} e^{A+B} \leq \operatorname{tr}\left(e^{A} e^{B}\right)$. This inequality is a basic tool in quantum statistical mechanics and extensions to infinite dimension have an extensive literature $[5,6]$. In this paper, we extend the classical Golden-Thompson theorem to normal matrices.

Throughout this paper, we adopt the following notation. Let $M_{n}$ be the set of all $n \times n$ complex matrices. For a matrix $A \in M_{n}$, as usual, its conjugate transpose is denoted by $A^{*}$. A matrix $A$ is called Hermitian if $A=A^{*}$, normal if $A^{*} A=A A^{*}$, and unitary if $A^{*} A=A A^{*}=I_{n}\left(I_{n}\right.$ is the identity matrix of order $\left.n\right)$. Given a matrix $A \in M_{n}$, the eigenvalues and singular values of $A$ are denoted by $\lambda_{1}(A), \ldots, \lambda_{n}(A)$, and $s_{1}(A), \ldots, s_{n}(A)$, respectively, where $\left|\lambda_{1}(A)\right| \geq \cdots \geq\left|\lambda_{n}(A)\right|$ and $s_{1}(A) \geq \cdots \geq s_{n}(A)$. In particular, when $A$ is positive semidefinite $(A \geq 0)$, then $\lambda_{1}(A) \geq \cdots \geq \lambda_{n}(A) \geq 0$. For simplicity, we denote $\lambda(A) \equiv\left(\lambda_{1}(A), \ldots, \lambda_{n}(A)\right)$ and $s(A) \equiv\left(s_{1}(A), \ldots, s_{n}(A)\right)$. Recall that the singular values of a matrix $A \in M_{n}$ are defined to be the eigenvalues of $|A| \equiv\left(A^{*} A\right)^{1 / 2}$, i.e., $s(A)=\lambda(|A|)$. Here $s_{1}(A)=\|A\|$ is the spectral norm of $A$. It is known that the spectral norm $\|\cdot\|$ over $M_{n}$ is unitarily invariant, i.e., $\|U A V\|=\|A\|$ for all unitary matrices $U, V$.

We now recall the concept of majorization (details can be found in [7-9]). We have the following basic majorant relations. For real vectors $x=\left(x_{1}, \ldots, x_{n}\right), y=\left(y_{1}, \ldots, y_{n}\right)$ in coordinates in decreasing order, we say that $x$ is weakly majorized by $y$, denoted by $x \prec_{w} y$, if

$$
\sum_{j=1}^{k} x_{j} \leq \sum_{j=1}^{k} y_{j}, \quad k=1, \ldots, n,
$$

and the weak log-majorant relation $x \prec_{\text {wlog }} y$ means

$$
\prod_{j=1}^{k} x_{j} \leq \prod_{j=1}^{k} y_{j}, \quad k=1, \ldots, n .
$$

O2014 Li and Zhao; licensee Springer. This is an Open Access article distributed under the terms of the Creative Commons Attribution License (http://creativecommons.org/licenses/by/2.0), which permits unrestricted use, distribution, and reproduction in any medium, provided the original work is properly cited. 
If in addition to $x \prec_{\text {wlog }} y, \prod_{1}^{n} x_{j}=\prod_{1}^{n} y_{j}$ holds, we say that $x$ is $\log$-majorized by $y$, denoted briefly by symbols $x \prec_{\log } y$. The following statement (see [8, 10]) is well known: $x \prec_{\text {wlog }} y$ yields $x \prec_{w} y$ for vectors $x, y \in R_{+}^{n}$.

Remark 1.1 For $x=\left(x_{1}, \ldots, x_{n}\right)$, we denote $|x|=\left(\left|x_{1}\right|, \ldots,\left|x_{n}\right|\right)$. Weyl's majorant theorem [10] says that $|\lambda(A)| \prec_{\log } s(A)$ for $A \in M_{n}$, that is,

$$
\left(\left|\lambda_{1}(A)\right|, \ldots,\left|\lambda_{n}(A)\right|\right) \prec_{\log }\left(\left|s_{1}(A)\right|, \ldots,\left|s_{n}(A)\right|\right) .
$$

The formula above implies that $|\lambda(A)| \prec_{w} s(A)$.

\section{Lemmas}

In this section, we shall propose some lemmas, laying the foundations of our main results in the next section.

Lemma 2.1 [11] If $A, B$ are positive semidefinite matrices, then

$$
\|A B\|^{t} \leq\left\|A^{t} B^{t}\right\|, \quad \text { and } \quad \lambda_{1}^{t}(A B) \leq \lambda_{1}\left(A^{t} B^{t}\right), \quad \text { for } t \geq 1 .
$$

Here note that $\|X\|=s_{1}(X)$ is the spectral norm of $X$.

Lemma 2.2 If $A, B \in M_{n}$ are normal matrices, then for any integer $m \geq 1$

$$
\|A B\|^{m} \leq\left\|\left|A^{m}\right| \cdot|B|^{m}\right\|=\left\|A^{m} B^{m}\right\| .
$$

Proof Take the polar decompositions $A=U|A|$ and $B=V|B|$. Here $U, V$ are unitary matrices. Since $A, B$ are normal, we can derive that $U|A|=|A| U$ and $V|B|=|B| V$ (see [12, 13]). Thus

$$
A B=U|A||B| V, \quad A^{m} B^{m}=U^{m}|A|^{m}|B|^{m} V^{m} .
$$

Since the norm $\|\cdot\|$ is unitary invariant, we obtain the following:

$$
\|A B\|^{m}=\|U(|A| \cdot|B|) V\|^{m}=\|(|A| \cdot|B|)\|^{m},
$$

and

$$
\left\|A^{m} B^{m}\right\|=\left\|U^{m} \cdot|A|^{m} \cdot|B|^{m} \cdot V^{m}\right\|=\left\||A|^{m} \cdot|B|^{m}\right\| .
$$

From Lemma 2.1, $\||A| \cdot|B|\|^{m} \leq\left\|\left(|A|^{m} \cdot|B|^{m}\right)\right\|$, we therefore conclude that

$$
\|A B\|^{m} \leq\left\|A^{m} B^{m}\right\| .
$$

Lemma 2.3 If $A, B \in M_{n}$ are normal matrices, then

$$
\|A B\|^{2}=s_{1}^{2}(|A| \cdot|B|)=\lambda_{1}\left(|A|^{2} \cdot|B|^{2}\right) .
$$


Proof Since $A$ and $B$ are normal, it follows from Lemma 2.2 that $\|A B\|=\||A| \cdot|B|\|$. So we get

$$
\|A B\|^{2}=\|(|A| \cdot|B|)\|^{2}=s_{1}^{2}(|A| \cdot|B|)=\lambda_{1}\left((|A| \cdot|B|)^{*} \cdot(|A| \cdot|B|)\right),
$$

as desired.

Lemma 2.4 If $A, B \in M_{n}$ are normal matrices, then for integers $m \geq 2$

$$
s_{1}^{m}(A B)=\|A B\|^{m} \leq \lambda_{1}\left(|A|^{m} \cdot|B|^{m}\right) .
$$

Proof By Lemma 2.3, we have $\|A B\|^{2}=\lambda_{1}\left(|A|^{2} \cdot|B|^{2}\right)$, and

$$
\|A B\|^{m}=\left(\|A B\|^{2}\right)^{m / 2}=\lambda_{1}^{m / 2}\left(|A|^{2}|B|^{2}\right) .
$$

Applying Lemma 2.1 to the right side above, we have the following:

$$
\lambda_{1}^{m / 2}\left(|A|^{2} \cdot|B|^{2}\right) \leq \lambda_{1}\left(|A|^{m} \cdot|B|^{m}\right) .
$$

Thus we get $\|A B\|^{m} \leq \lambda_{1}\left(|A|^{m} \cdot|B|^{m}\right)$ for integers $m \geq 2$, as desired.

Here we note that $\left|A^{m}\right|=|A|^{m}$ holds for any normal matrix.

The following lemma needs the notion of the Grassmann power $\Lambda^{k} A$ (or antisymmetric tensor product), which can be found in [8, p.18].

Lemma 2.5 If $A \in M_{n}, 1 \leq k \leq n$, then for any natural number $m$, the following holds:

$$
\prod_{j=1}^{k} s_{j}\left(A^{m}\right) \leq \prod_{j=1}^{k} s_{j}^{m}(A), \quad \text { and } \quad \prod_{j=1}^{n} s_{j}\left(A^{m}\right)=\prod_{j=1}^{n} s_{j}^{m}(A) .
$$

i.e.,

$$
s\left(A^{m}\right) \prec_{\log } s^{m}(A) .
$$

Proof For $1 \leq k \leq n$, consider the $k$ th antisymmetric tensor product $\Lambda^{k} A$ of $A \in M_{n}$. It is known [8, p.18] that $\Lambda^{k}\left(A^{m}\right)=\left(\Lambda^{k} A\right)^{m}$ and

$$
s_{1}\left(\Lambda^{k}\left(A^{m}\right)\right)=s_{1}\left(\left(\Lambda^{k} A\right)^{m}\right)=\left\|\left(\Lambda^{k} A\right)^{m}\right\| \leq\left\|\Lambda^{k} A\right\|^{m}=\left(s_{1}\left(\Lambda^{k} A\right)\right)^{m} .
$$

Thus

$$
\prod_{j=1}^{k} s_{j}\left(A^{m}\right) \leq \prod_{j=1}^{k} s_{j}^{m}(A), \quad k=1, \ldots, n .
$$

In particular,

$$
\prod_{j=1}^{n} s_{j}\left(A^{m}\right)=\prod_{j=1}^{n} s_{j}^{m}(A)=|\operatorname{det}(A)|^{m}
$$

which, equivalently, says that $s\left(A^{m}\right) \prec_{\log } s^{m}(A)$. This completes the proof. 


\section{Main results}

In this section, we shall present the main results of this paper.

Theorem 3.1 If $A, B \in M_{n}$ are normal matrices, then

$$
s\left(e^{A+B}\right) \prec \log \lambda\left(\left|e^{A}\right| \cdot\left|e^{B}\right|\right) .
$$

Proof Let $A, B \in M_{n}$ be normal matrices. It is clear that $\Lambda^{k} e^{A / m}, \Lambda^{k} e^{B / m}$ are normal for $1 \leq k \leq n$. By replacing $A, B$ by $\Lambda^{k} e^{A / m}, \Lambda^{k} e^{B / m}$ in Lemma 2.4, respectively, we can obtain the following for integers $m \geq 2$ :

$$
\begin{aligned}
s_{1}^{m}\left(\Lambda^{k}\left(e^{A / m} e^{B / m}\right)\right) & =s_{1}^{m}\left(\Lambda^{k} e^{A / m} \Lambda^{k} e^{B / m}\right) \\
& \leq \lambda_{1}\left(\left|\Lambda^{k} e^{A}\right|\left|\Lambda^{k} e^{B}\right|\right)=\lambda_{1}\left(\Lambda^{k}\left(\left|e^{A}\right|\left|e^{B}\right|\right)\right) .
\end{aligned}
$$

Here we note that $\left|\Lambda^{k} A\right|=\Lambda^{k}|A|$ because $\left|\Lambda^{k} A\right|^{2}=\Lambda^{k}|A|^{2}$. So we obtain

$$
\prod_{j=1}^{k} s_{j}^{m}\left(e^{A / m} e^{B / m}\right) \leq \prod_{j=1}^{k} \lambda_{j}\left(\left|e^{A}\right| \cdot\left|e^{B}\right|\right) .
$$

From Lemma 2.5, we have

$$
\prod_{j=1}^{k} s_{j}\left[\left(e^{A / m} e^{B / m}\right)^{m}\right] \leq \prod_{j=1}^{k} s_{j}^{m}\left(e^{A / m} e^{B / m}\right) .
$$

Thus,

$$
\prod_{j=1}^{k} s_{j}\left[\left(e^{A / m} e^{B / m}\right)^{m}\right] \leq \prod_{j=1}^{k} \lambda_{j}\left(\left|e^{A}\right|\left|e^{B}\right|\right) .
$$

The Lie product formula $[8, \mathrm{p} .254]$ says that for any matrices $A, B$

$$
\lim _{m \rightarrow \infty}\left(e^{A / m} e^{B / m}\right)^{m}=e^{A+B} .
$$

Thus taking $m \rightarrow \infty$ in the inequality above yields

$$
\prod_{j=1}^{k} s_{j}\left(e^{A+B}\right) \leq \prod_{j=1}^{k} \lambda_{j}\left(\left|e^{A}\right|\left|e^{B}\right|\right) .
$$

Finally we note that

$$
\prod_{j=1}^{n} s_{j}\left(e^{A+B}\right)=\left|\operatorname{det}\left(e^{A+B}\right)\right|=\left|\operatorname{det}\left(e^{A} e^{B}\right)\right|=\prod_{j=1}^{n} \lambda_{j}\left(\left|e^{A}\right|\left|e^{B}\right|\right) .
$$

Thus we get

$$
s\left(e^{A+B}\right) \prec \log \lambda\left(\left|e^{A}\right| \cdot\left|e^{B}\right|\right) .
$$

This completes the proof. 
From Theorem 3.1, we know that

$$
s\left(e^{A+B}\right) \prec \log \lambda\left(\left|e^{A}\right| \cdot\left|e^{B}\right|\right)
$$

On the other hand, the following equation holds:

$$
\lambda\left(\left|e^{A}\right| \cdot\left|e^{B}\right|\right)=\lambda\left(\left|e^{A}\right|^{1 / 2} \cdot\left|e^{B}\right| \cdot\left|e^{A}\right|^{1 / 2}\right)=s\left(\left|e^{A}\right|^{1 / 2} \cdot\left|e^{B}\right| \cdot\left|e^{A}\right|^{1 / 2}\right) .
$$

The above two inequalities yield the following:

$$
s\left(e^{A+B}\right) \prec_{\log } s\left(\left|e^{A}\right|^{1 / 2} \cdot\left|e^{B}\right| \cdot\left|e^{A}\right|^{1 / 2}\right) .
$$

Thus, we can get the following corollary by using the Fan Dominance Principle [10, p.56].

Corollary 3.2 If $A, B \in M_{n}$ are normal matrices, then

$$
\left\|\left|e^{A+B}\right|\right\| \leq\left\|\left.|| e^{A}\right|^{1 / 2} \cdot\left|e^{B}\right| \cdot\left|e^{A}\right|^{1 / 2} \mid\right\|
$$

for all unitarily invariant norms $\||\cdot|\|$.

From Theorem 3.1, we can also have the following result.

Theorem 3.3 If $A, B \in M_{n}$ are normal matrices, then

$$
\left|\lambda\left(e^{A+B}\right)\right| \prec \log \lambda\left(\left|e^{A}\right| \cdot\left|e^{B}\right|\right) .
$$

Proof By Weyl's majorant theorem we have $|\lambda(A)| \prec_{\log } s(A)$. Hence Theorem 3.1 implies the desired inequality in Theorem 3.3.

Note that Theorem 3.3 strengthens the Golden-Thompson inequality:

$$
\left|\operatorname{tr}\left(e^{A+B}\right)\right| \leq \operatorname{tr}\left(e^{A} e^{B}\right)
$$

for Hermitian matrices $A, B$.

Theorem 3.4 If $A, B$ are normal matrices, then

$$
\left|\operatorname{tr}\left(e^{A+B}\right)\right| \leq \operatorname{tr}\left(\left|e^{A}\right| \cdot\left|e^{B}\right|\right)
$$

Proof Because $x \prec_{\log } y$ implies $x \prec_{w} y$, it follows from Theorem 3.3 that

$$
\left|\lambda\left(e^{A+B}\right)\right| \prec_{w} \lambda\left(\left|e^{A}\right| \cdot\left|e^{B}\right|\right) .
$$

Taking the traces above, we have

$$
\left|\operatorname{tr}\left(e^{A+B}\right)\right| \leq \operatorname{tr}\left(\left|e^{A}\right| \cdot\left|e^{B}\right|\right)
$$

So we get the desired inequality. This completes the proof. 
Of course, Theorem 3.4 is an extension of Golden-Thompson inequality:

$$
\operatorname{tr}\left(e^{A+B}\right) \leq \operatorname{tr}\left(e^{A} e^{B}\right)
$$

\section{for Hermitian matrices $A, B$.}

\section{Competing interests}

The authors declare that they have no competing interests.

\section{Authors' contributions}

$\mathrm{HL}$ carried out the theorems and corresponding proofs, DZ checked the proofs carefully, and provided numerical examples and valuable suggestions. All authors read and approved the final manuscript.

\section{Acknowledgements}

The authors would like to thank the referees for reading this work carefully, providing valuable suggestions and comments, which have significantly improved this article. This work is supported by National Natural Science Foundation of China (Grant No. 61379001).

\section{Received: 5 July 2013 Accepted: 12 December 2013 Published: 09 Jan 2014}

\section{References}

1. Golden, S: Lower bounds for the Helmholtz function. Phys. Rev. 137, B1127-B1128 (1965)

2. Hiai, F: Equality cases in matrix norm inequalities of Golden-Thompson type. Linear Multilinear Algebra 35, 239-249 (1994)

3. Symanzik, K: Proofs and refinements of an inequality of Feynman. J. Math. Phys. 6, 1155-1156 (1965)

4. Thompson, CJ: Inequality with applications in statistical mechanics. J. Math. Phys. 6, 1812-1813 (1965)

5. Araki, H: Golden-Thompson and Peierls-Bogoliubov inequalities for a general von Neumann algebra. Commun. Math. Phys. 34, 167-178 (1973)

6. Ruskai, MB: Inequalities for traces on von Neumann algebras. Commun. Math. Phys. 26, $280-289$ (1972)

7. Ando, T: Majorizations, doubly stochastic matrices, and comparison of eigenvalues. Linear Algebra Appl. 118, 163-248 (1989)

8. Bhatia, R: Matrix Analysis. Springer, Berlin (1997)

9. Marshall, AW, Olkin, I: Inequalities: Theory of Majorization and Its Applications. Academic Press, New York (1979)

10. Zhan, X: Matrix Inequalities. Lecture Notes in Mathematics, vol. 1790. Springer, Berlin (2002)

11. Furuta, T: Norm inequalities equivalent to Lowner-Heinz theorem. Rev. Math. Phys. 1, 135-137 (1989)

12. Horn, RA, Johnson, CR: Matrix Analysis. Cambridge University Press, Cambridge (1985)

13. Zhang, F: Matrix Theory: Basic Results and Techniques. Universitext. Springer, New York (1999)

10.1186/1029-242X-2014-14

Cite this article as: $L i$ and Zhao: An extension of the Golden-Thompson theorem. Journal of Inequalities and Applications 2014, 2014:14

\section{Submit your manuscript to a SpringerOpen ${ }^{\ominus}$ journal and benefit from:}

- Convenient online submission

- Rigorous peer review

- Immediate publication on acceptance

Open access: articles freely available online

- High visibility within the field

- Retaining the copyright to your article 